\title{
ZU DEN SATZFORMEN IM DEUTSCHEN UND WIE SIE DURCH DIE VERBVALENZ BEEINFLUSST WERDEN
}

\section{O VORWORT}

Diesen Beitrag widme ich meinem Lehrer und Kollegen Prof. Dr. Siegfried Heusinger anlässlich seines Jubiläums. Siegfried Heusinger hat meine Diplom- und Magisterarbeit betreut und war auch mein Doktorvater. Er hat mich vor allem in die Geheimnisse der deutschen Syntax eingeweiht, die Fachdisziplin, die ich selbst später von ihm als Lehrer übernommen habe. Deshalb soll auch das Thema dieses Beitrags ein grundsätzlich syntaktisches sein und darüber hinaus didaktisch ausgerichtet. Es soll darin darauf hingewiesen werden, dass auch elementare syntaktische Fragen auf Probleme stoßen können und dass es daher besonders wichtig ist, wie man diese Probleme zu didaktischen Zwecken überbrückt.

\section{ZUR KLASSIFIKATION DER SATZFORMEN: EINFACHER SATZ UND ZUSAMMENGESETZTER SATZ}

Mit Bezug auf die Satzformen kann man einfache Sätze (= Einfachsätze) und zusammengesetzte Sätze (= komplexe Sätze) unterscheiden. Das Kriterium für die Unterscheidung dieser Satzformen ist der Aufbau des Satzes als einer Konstruktion, die zwischen zwei Interpunktionszeichen steht und mit einer Majuskel beginnt. ${ }^{1}$ Diese Konstruktion kann auch mit dem Oberbegriff „Ganzsatz" bezeichnet werden, egal wie ihre Form ist (vgl. Eroms 2000: 53).

\subsection{Einfacher Satz}

Wenn in einer Satzstruktur nur ein finites Prädikat steht, haben wir es mit einem einfachen Satz zu tun:

\section{Anja lebt in Ljubljana.}

Das finite Prädikat ist ein Ausdrucksmittel der Prädikation. Die Prädikation ist die sprachliche Kategorie, mit deren Hilfe im Satz über das Handeln, den Zustand, über

stojan.bracic@ff.uni-lj.si

1 Bei der Bestimmung der Formen von Nebensätzen gilt jedoch ein anderes Kriterium, und zwar die Art, wie sich der Nebensatz an den Hauptsatz anschließt (uneingeleitet; eingeleitet: Subjunktionalsatz, Relativsatz, indirekter Fragesatz). 
Eigenschaften oder den Status des zumeist durch das Subjekt bezeichneten Referenzgegenstandes etwas ausgesagt wird:

$$
(\mathrm{P} \leftarrow \mathrm{R}) \operatorname{Anja}(\leftarrow \mathrm{PR}) \operatorname{lebt}(\mathrm{P}) \text { in Ljubljana. }
$$

(QŁZu unterscheiden ist also zunächst einmal zwischen den Kategorien Referenz $(\mathrm{R})$ und Prädikation (PR), außerdem zwischen der Kategorie Prädikation und dem Prädikat (P) als einem Ausdrucksmittel der Prädikation.

\subsection{Zusammengesetzter Satz}

Zusammengesetzte Sätze sind Sätze, in denen neben einem Einfachsatz mit einem finiten Prädikat mindestens ein weiteres finites Prädikat oder mindestens ein anderes explizites $^{2}$ Ausdrucksmittel der Prädikation steht. Der zusammengesetzte Satz als Ganzsatz besteht somit aus mindestens zwei Teilsätzen, und zwar entweder aus zwei Hauptsätzen oder aus einem Hauptsatz und einem Nebensatz:

\section{Es regnet(,) und die Blumen blühen.}

Peter weiß, dass Anja ihn besucht.

Im ersten Fall haben wir die sog. Parataxe (die Satzreihe) - Nebenordnung von gleichwertigen Teilsätzen. ${ }^{3}$ Im zweiten Fall haben wir die sog. Hypotaxe (das Satzgefüge) - mindestens ein Nebensatz als Teilsatz ist dem anderen Teilsatz, dem Hauptsatz, untergeordnet. Der Nebensatz kann daher nicht allein stehen:

\section{Dass Anja ihn besucht.}

Aber auch nicht jeder Hauptsatz ist selbstständig. Jene Hauptsätze, die mit einem Nebensatz als Sonderform eines valenzmäßig obligatorisch notwendigen Satzgliedes ein Satzgefüge bilden, sind nicht selbstständig. Vgl. das Satzgefüge mit einem Prädikativsatz und das Satzgefüge mit einem (obligatorischen) Objektsatz:

Du bist, was du isst.

*Du bist.

Ich weiß, dass sie kommt.

*Ich weiß.

2 Explizite Ausdrucksmittel der Prädikation sind finite und infinite Verbformen. Implizite Ausdrucksmittel der Prädikation sind hingegen Nominalisierungen (z. B. Hilfe $\leftarrow$ helfen), Attribuierungen (z. B. Eine grüne Blume $\leftarrow$ die Blume ist grün) und Wortbildungen (z. B. Abendlied $\leftarrow$ ein Lied, das abends gesungen wird).

3 Von der Parataxe ist der zusammengezogene Satz zu unterscheiden, der vorkommt, wenn in einer Parataxe mindestens ein identisches Element (Satzglied) und mindestens zwei verschiedene Elemente (Satzglieder) vorkommen und das identische Element analeptisch ausgelassen wird: Anja studiert fleißig für die Prüfung und hilft ihren Eltern bei der Arbeit auf dem Bauernhof (vgl. Helbig/Buscha 1998: 650). 
Im zusammengesetzten Satz kann aber anstatt eines Nebensatzes mit einem zweiten finiten Prädikat auch eine Konstruktion stehen, die einem solchen Nebensatz funktional äquivalent ist. Es gibt zwei solche Konstruktionen, das sind die Infinitivkonstruktion und die Partizipialkonstruktion.

\section{Er bittet mich(,) ihm zu helfen. \\ Nach Hause gehend(,) sangen sie schöne Lieder.}

Weil Infinitivkonstruktionen und Partizipialkonstruktionen mit Nebensätzen syntaktisch-funktional gleichwertig sind, können sie Nebensätze (unter gewissen Bedingungen) ersetzen.

Sie können aber - genauso wie Nebensätze - nicht allein einen selbstständigen Einfachsatz bilden.

*Ihm zu helfen.

*Nach Hause gehend.

\subsection{Satzperiode}

Zusammengesetzte Sätze können aus zwei Teilsätzen oder - was häufiger der Fall ist - aus mehreren Teilsätzen gebaut sein. Bei Satzperioden geht es jedoch um noch kompliziertere, mehrfach zusammengesetzte Sätze, die entweder nur parataktisch oder - zumeist - hypotaktisch gebaut sind. Es gibt allerdings kein exaktes quantitatives Kriterium, aus wie vielen Teilsatzkonstituenten ein komplexer Satz zusammengesetzt werden muss, um (bereits) von einer Periode sprechen zu können.

Ulla Fix leitet ihren Beitrag zur Stilanalyse als Mittel der Erziehung zum Wiederspruch (1992: 145) mit einem Zitat aus Adornos „Erziehung zur Mündigkeit“ (1969) ein:

Ich würde auf die Gefahr hin, daß Sie mich einen Philosophen schelten, der ich nun einmal bin, sagen, daß die Gestalt, in der Mündigkeit sich heute konkretisiert, die ja gar nicht ohne weiteres vorausgesetzt werden kann, weil sie an allen, aber wirklich an allen Stellen unseres Lebens überhaupt erst herzustellen wäre, daß also die einzige Konkretisierung der Mündigkeit darin besteht, daß die paar Menschen, die dazu gesonnen sind, mit aller Energie darauf hinwirken, daß die Erziehung eine Erziehung zum Widerspruch und zum Widerstand ist.

Für die Satzform ist somit nicht die durch die Wortanzahl bestimmte Satzlänge maßgeblich, sondern die Anzahl der expliziten prädikativen Einheiten.

Ein Einfachsatz kann demnach (viel) länger sein als ein zusammengesetzter Satz. Im Folgenden wird einem zusammengesetzten Satz mit nur vier Wörtern und vier Satzgliedern (Subjekt und Prädikat des Hauptsatzes, Subjekt und Prädikat des Nebensatzes) ein einfacher Satz mit dreißig Wörtern, aber mit nur drei Satzgliedern, und zwar dem Subjekt, dem Prädikat und dem Prädikativ (mit dazugehörigen Attributen) gegenübergestellt. 
Zusammengesetzter Satz:

Ich weiß, sie kommt.

Einfachsatz:

Der ultimative Sieg über die schwer dezimierten sowjetischen Streitkräfte und die Nutzung der dabei erbeuteten Rohstoffe und Ressourcen zur Niederwerfung aller verbleibenden Gegner waren das erklärte Ziel der „Operation Blau“."

\section{EINFACHER SATZ UND DIE VERBVALENZ}

Der Einfachsatz zeigt sich in mehreren Erscheinungsformen. Die Subklassifizierung der Formen des einfachen Satzes kann man nur verstehen, wenn man den Begriff der Verbvalenz heranzieht. Die Verbvalenz ist die Eigenschaft des Verbs, um sich herum freie Stellen/Lücken zu eröffnen, die im Normalfall durch verschiedene Satzglieder/ Mitspieler/Aktanten/Argumente/Komplemente geschlossen werden (müssen). Man spricht mit Helbig/Schenkel (1982: 33) vom Stellenplan des Verbs, der sowohl quantitativ (Anzahl der Mitspieler) als qualitativ (Art der Mitspieler) bestimmbar ist.

Das Verb hören ist z. B. im Satz

Peter hört Musik.

zweiwertig, weil es gemäß seinem valenzbedingten/valenzabhängigen/valenzgeprägten Stellenplan in einem Satz mit zwei Aktanten stehen muss: mit einem Subjekt/ $\mathrm{Su}$ (wer hört was/wen?) $)^{5}$ und mit einem Akkusativobjekt (O4) (was/wen hört jemand?). Schematisch lässt sich das, wie folgt, veranschaulichen:

$\begin{array}{lll}\text { Peter } & \text { hört/ } & \text { Musik. } \\ \text { Wer? }(\mathrm{Su}) & & \text { Was? }(\mathrm{O} 4)^{6}\end{array}$

Die Aktanten, die im Stellenplan des Verbs verankert sind, sind notwendige Aktanten. Sie sind durch die semantische Struktur des Verbs (durch dessen Semem) prädestiniert. Das Semem des Verbs hören setzt z. B. zwei Seme (semantische Merkmale) voraus: einerseits den Träger der Handlung, genauer, des physiologischen Prozesses (Agens - Subjekt) und andererseits das physikalische Phänomen, das akustisch

4 Aus: Mascolo (2010: 80).

5 Fragestellungen sind dabei ein geeignetes operationales Hilfsmittel.

6 Der Valenzbegriff ist durch Tesnière als metaphorische Bezeichnung aus der Chemie in die Linguistik übernommen worden. Analog dazu kann z. B. ein Wassermolekül $\left(\mathrm{H}_{2} 0\right)$, in dem sich ein divalentes Sauerstoffatom mit zwei monovalenten Wasserstoffatomen verbindet, folgendermaßen skizziert werden:

$\mathrm{H}-\mathrm{O}-\mathrm{H}$ 
wahrgenommen wird (Patiens - Akkusativobjekt). ${ }^{7}$ Oder mit Worten von Fandrych/ Thurmair: „Verben [...] bilden das Organisationszentrum von Sätzen. [...] Von ihrer Bedeutung her kann man mit Verben Ereignisse in Szene setzen.“ (2018: 193)

Bei notwendigen Aktanten kann man mit Helbig/Schenkel (1982: 33; 46) jedoch zwei Arten unterscheiden: obligatorisch notwendige und fakultativ notwendige Aktanten. Obligatorisch notwendige Aktanten sind diejenigen notwendigen Aktanten, die nicht weglassbar sind. Das bedeutet, dass ein Satz ohne sie ungrammatisch wird. Das dreiwertige Verb legen hat beispielsweise in seinem Stellenplan drei Aktanten, die obligatorisch sind. Lässt man nur einen Mitspieler weg, wird der Satz ungrammatisch.

Anton legt das Buch in ein falsches Fach.

* Legt in ein falsches Fach.

*Anton legt in ein falsches Fach.

*Anton legt das Buch.

Fakultativ notwendige Aktanten sind auf der anderen Seite solche Aktanten, die zwar durch entsprechende Seme im Stelleplan des Verbs enthalten sind, die aber unter Umständen weglassbar sind.

Das Verb einsteigen ist beispielsweise zweiwertig:

\section{Anja steigt in die Straßenbahn ein.}

Es steht mit einem Subjekt/Su (Wer steigt ein?) und mit einer Lokalbestimmung/ LB (Wohin/wo steigt jemand ein?). Schematisch lässt sich das wiederum, wie folgt, veranschaulichen:

$\begin{array}{lll}\text { Anja } & \text { /steigt ein/ } & \text { in die Straßenbahn } \\ \text { wer? }(\mathrm{Su})- & \mathrm{P} & - \text { wohin? }(\mathrm{LB})\end{array}$

Die von der Verbvalenz erforderlichen Aktanten/Mitspieler können also in zwei Unterarten unterteilt werden.

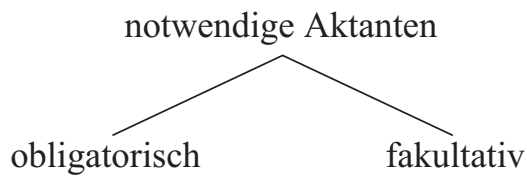

Es erhebt sich die Frage, unter welchen Umständen die Lokalbestimmung als fakultativer Aktant fehlen darf? Das ist nur dann möglich, wenn entweder aus dem Kotext (schriftliche Kommunikation) oder aus der kommunikativen Situation (mündliche Kommunikation) hervorgeht und sowohl für den Sprecher/Schreiber als auch für den

7 Das Verb hören kann allerdings auch als einwertiges Verb verwendet werden und dann bedeuten, dass jemand hören kann, also gesundes Hörvermögen hat, nicht taub ist (vgl. Unterkapitel 4). 
Leser/Hörer (gemeinsames Wissen; „das geteilte Wissen“ in Wöllstein 2016: 1230) eindeutig ist, wohin jemand einsteigt. Die Nennung der Lokalbestimmung kann man sich im konkret realisierten Satz - auf der sichtbaren oder hörbaren Textoberfläche aus sprachökonomischen Gründen sparen:

Die Realisierung eines fakultativen Aktanten ist von pragmatischen Faktoren (Sprecher-/Hörerorientiertheit), jedoch nicht von der (Satz)Konstruktionsspezifik abhängig. In dem Sinne hat der Sprecher/Schreiber die Wahlfreiheit, sie zu realisieren, was für obligatorische Aktanten nicht der Fall ist. (Krevs Birk 2006: 75)

Im Unterschied zur Valenz des zweiwertigen Verbs hören, dass mit zwei obligatorisch notwendigen Elementen steht, lässt sich die Valenz des Verbs einsteigen mit Helbig/Schenkel (1982) nummerisch so ausdrücken:

einsteigen: $\quad 1+(1)=2$

Das besagt: Das Verb einsteigen ist insgesamt zweiwertig, dessen (Gesamt)wertigkeit 2 ist die Summe von einem obligatorischen und einem fakultativen Aktanten (letzterer steht daher in Klammern).

Vor diesem Hintergrund kann man grob gesehen zwei Formen des Einfachsatzes unterscheiden: einen reinen einfachen Satz und das syntaktische Minimum/das Satzminimum (Helbig/Schenkel 1982: 33; Jung 1990: 32). Den reinen einfachen Satz bilden das Prädikat und alle notwendigen (= obligatorischen und fakultativen) Aktanten, sodass also die Satzstruktur dem Stellenplan des Verbs voll gerecht wird. Das syntaktische Minimum haben wir in unserem Fall jedoch dann, wenn das fakultative Element entfällt.

Anja steigt $\quad O \quad$ ein.

Diese Satzform heißt syntaktisches Minimum oder Satzminimum, weil sie die minimale Form des Satzes ist, die noch grammatisch richtig/vollkommen/akzeptabel ist.

Von dem reinen einfachen Satz kommt man zum syntaktischen Minimum dadurch, dass fakultativ notwendige Satzglieder, falls solche überhaupt vorhanden sind, gestrichen/eliminiert/ausgeblendet werden. Syntaktisches Minimum ist also - mathematisch gesehen - reiner einfacher Satz minus fakultative Aktanten.

Würden wir im so entstandenen syntaktischen Minimum weitere Elemente streichen/ tilgen/weglassen/eliminieren, würden wir die Grenze der Grammatikalität des Satzes verletzen: Wir hätten es mit einer nicht mehr grammatisch richtigen (= ungrammatischen) Struktur zu tun (die außerdem leicht mit einem Befehlssatz vermengt werden könnte):

*Steigt ein.

Daraus kann man schlussfolgern: Bei denjenigen Verben, die nur obligatorische Aktanten (also keine fakultativen Aktanten) im Stellenplan enthalten (z. B. legen, wohnen, 
dauern), ist reiner einfacher Satz zugleich schon syntaktisches Minimum selbst. Während das Verb hören mit zwei obligatorischen Aktanten steht (s. oben), erfordert die Valenz des Verbs legen gar 3 obligatorisch notwendige Aktanten: legen (legen $3+(0)=3)($ s. oben).

Das Experiment, mit dem man gewisse (= fakultativ notwendige) Elemente einer bestehenden Satzstruktur eliminiert, um sich an die Grenze der Grammatikalität und Verständlichkeit dieser Satzstruktur heranzutasten, heißt Weglassprobe oder Eliminierungstest (Helbig/Schenkel 1982: 33).

Auf der anderen Seite kann man aber den reinen einfachen Satz bis zu einer vernünftigen Grenze (Problem der Rezeption) auch erweitern, und zwar mit sog. freien Angaben. Das sind Elemente, die nicht im Stellenplan des Verbs stehen, die also von der Verbvalenz nicht gefordert sind. In diesem Fall spricht man vom sogenannten erweiterten einfachen Satz.

Anja steigt jeden Morgen an dieser Haltestelle in die Straßenbahn ein.

Anja steigt (jeden Morgen) (an dieser Haltestelle) in die Straßenbahn ein.

In Analogie zur Weglassprobe spricht man in diesem Fall von der sog. Erweiterungsprobe (Wöllstein 2016: 131). Auch diese Probe hat einen didaktischen Wert, weil sie den Studierenden Gelegenheit bietet, nebenbei über beliebig viele Ergänzungsmöglichkeiten ihre lexikalische Kompetenz zu vertiefen.

Fassen wir zusammen: Es sind folgende Varianten des einfachen Satzes zu unterscheiden:

reiner einfacher Satz: obligatorische und fakultative (= allesamt notwendige) Aktanten

syntaktisches Minimum: (nur) obligatorische Aktanten

erweiterter einfacher Satz: obligatorische Aktanten + fakultative Aktanten + freie Angaben

Tabelle: Verbvalenz und Satzformen

\begin{tabular}{|l|l|}
\hline reiner einfacher Satz & Anja steigt in die Straßenbahn ein. \\
\hline syntaktisches Minimum/Satzminimum & Anja steigt ein. \\
\hline erweiterter einfacher Satz & $\begin{array}{l}\text { Anja steigt jeden Morgen an dieser } \\
\text { Haltestelle in die Straßenbahn ein. }\end{array}$ \\
\hline
\end{tabular}

Schema: Satzformen

Ganzsatz

$\begin{array}{lc}\text { einfacher Satz } & \text { zusammengesetzter Satz } \\ \text { erweiterter einfacher Satz } & \text { Parataxe } \\ \text { reiner einfacher Satz } & \text { (zusammengezogener Satz) } \\ \text { Satzminimum } & \text { Hypotaxe } \\ \text { (Ellipse) } & \text { Satzperiode }\end{array}$


Die Ellipse

Wenn im obigen Schema zu den Formen des (Ganz)satzes um der Anschaulichkeit willen versucht wird, von links nach rechts hin von einfacheren zu komplexeren Satzstrukturen überzugehen, so ließe sich spiegelbildlich zur rechtspositionierten Satzperiode als der kompliziertesten Satzstruktur deren Pendant, nämlich die Ellipse, ganz links anbringen. Die Ellipse kommt vor, wenn (in gewissen kommunikativen Situationen) sich sogar gegen das syntaktische Minimum insoweit verstoßen lässt, als man eine grammatisch unvollständige Satzkonstruktion ohne finites Prädikat (in diesem Fall auch Setzung genannt (vgl. Greule 2018: 5) oder ohne Subjekt oder ohne einen anderen obligatorischen Aktanten bekommt. Ellipsen sind jedoch kommunikativ akzeptabel, weil sie verschiedene kommunikative Aufgaben erfüllen können. Häufig sind sie in emotional gefärbten Ausrufen oder in unechten Fragen (Gegenfragen) vorhanden.

Sofort! Keine Ahnung! Feuer! Hilfe! Warum denn nicht ${ }^{8}$

\section{VERBALE POLYSEMIE UND SATZFORMEN}

Die Valenz des jeweiligen Verbs ist, wie oben angedeutet (2), eng mit seiner Semantik verbunden. Deshalb muss bei jedem polysemen Verb für jedes Semem die Valenz gesondert bestimmt werden. Die Valenz des Verbs umziehen könnte man in Anlehnung an Helbig und Schenkel (1982), aber leicht vereinfacht, folgendermaßen veranschaulichen:

umziehen

Bedeutung 1:

quantitative Ebene:

'eine andere Wohnung nehmen'

morphologische Ebene:

$1+(1)=2$

obligatorischer Aktant: Sn (Substantiv im Nominativ) fakultativer Aktant: pS (Substantiv mit Präposition)

semantische Ebene:

Der Nachbar zieht um.

Der Betrieb zieht um.

Er zieht in einen Neubau um.

Sn ist hum (menschlich) oder abstrakt (aber menschlich gedacht); die Präposition ist in oder nach.
Bedeutung 2:
'sich anders kleiden"
quantitative Ebene:
$2+(0)=2$
morphologische Ebene: obligatorischer Aktant1: Sn
obligatorischer Aktant2: Sa (Substantiv im Akkusativ)

$8 \mathrm{Zu}$ unterscheiden sind jedoch von den Ellipsen die Aposiopesen. Letztere stellen Stilfiguren dar, bei denen nicht wie bei Ellipsen problemlos eine Ergänzung hinzufügbar ist, sondern mehrere potenzielle Fortsetzungen möglich sind (vgl. Fix 2018: 121). 
semantische Ebene (hum): Die Mutter zieht das Kind um.

Die Mutter zieht sich um.

Vor diesem Hintergrund kann man auch erklären, wie der Unterschied zwischen fakultativen Aktanten und freien Angaben ist. Beide können im konkreten Satz fehlen: freie Aktanten, weil deren semantische Informationen im konkreten Verbsemem mit keinem Sem vorgesehen sind, weil man sie also in der Regel nicht vermisst und beliebig hinzufügen oder weglassen kann, während fakultative Aktanten - wenn auch nicht immer explizit ausgedrückt - mitgedacht werden können bzw. müssen:

Der Nachbar zieht um. (Man weiß: etwa nach Maribor.)

Nur die semantischen Kasus Agens (Subjekt) und Lokativ (Adverbiale) sind als Seme im Semem des Verbs umziehen (Bedeutung 1) enthalten. Viele andere Informationen (wann, warum, wie, mit wem, auf welche Art, trotz welchen Umstandes ...) sind nicht satzkonsistent, sie sind nicht an die Bedeutung des Verbs immanent angeknüpft und können auch bei anderen Verben vorkommen. Der Satz Der Nachbar zieht um ist daher ein syntaktisches Minimum, der Satz Der Nachbar zieht nach Berlin um hingegen ein reiner einfacher Satz.

\section{VALENZMODIFIKATION UND SATZFORMEN}

Bei einigen Verben kommt es häufig zu einer semantischen Verschiebung/Modifikation, die mit einer Valenzreduktion einhergeht, z. T. auch im Sinne einer euphemistischen Ausdrucksweise, und solche Verben werden auf diesem Weg einwertig. Das Verb sitzen kann beispielsweise auch bedeuten 'im Gefängnis sitzen', 'eingesperrt sein $^{\prime}$, trinken kann auch bedeuten 'übermäßig trinken', 'alkoholsüchtig sein', rauchen kann auch die allgemeine Bedeutung haben 'Raucher sein'. ${ }^{10}$

Valenzreduziert können Verben auch sein, wenn die kommunikative Situation Aufschluss über die fehlenden semantischen Angaben gibt:

Das Faltblatt informiert über die Geschichte des Schlosses. (Nämlich die interessierte Leserschaft.)

Der Gastgeber empfängt in einem schönen Wohnzimmer. (Nämlich die Eingeladenen.)

Maribor ist $125 \mathrm{~km}$ entfernt. (Eine deiktische Schlussfolgerung ist aufgrund einer konkreten kommunikativen Situation möglich: z. B. von hier aus, wenn wir uns im Moment des Sprechens etwa in Ljubljana befinden.)

Pragmatisch erklärbar ist auch die Valenzreduktion von lernen im folgenden Textsegment:

9 Bekannt ist das humoristische Wortspiel Schon mancher hat gesessen, weil er gestanden hat.

10 Vgl. auch Fußnote 7. 
Seien Sie dankbar: "So beugen Sie dem Herzinfarkt vor"

Der Psychologe Glenn Afflec von der Universität von Connecticut befragte 287 Menschen nach einem Herzinfarkt. Dabei entdeckte er, dass diejenigen, die aus ihrer Krankheit gelernt haben, innerhalb der nächsten acht Jahre seltener einen weiteren Infarkt erleiden würden. (aus: Reader's Digest, 14. 7. 2017)

Trotz des fehlenden obligatorischen Aktanten zum Verb lernen kann man von keinerlei Ellipse sprechen, weil man ganz genau weiß: 'lernen, wie man gesünder leben muss'.

\section{SCHLUSS}

Bei den Satzformen muss man zwischen den Formen des Ganzsatzes und der Form des Nebensatzes unterscheiden. Im Ganzsatz ist das Kriterium für die Bestimmung der Satzform die Anzahl der explizit ausgedrückten Prädikationen, im Nebensatz als einem vom Hauptsatz abhängigen Teilsatz jedoch die Art, wie sich der Nebensatz an den Hauptsatz anschließt.

Der Ganzsatz ist dabei ein Oberbegriff, der für alle Satzkonstruktionen stehen kann, deren Erkennungsmerkmale eine Majuskel an deren Anfang und ein Interpunktionszeichen am Ende sind, und somit sowohl Einfachsätze als auch komplexe Sätze umfasst.

Der Einfachsatz kann mit viel sprachlichem Material gebaut und daher lang sein. Das ist der Fall im Nominalstil, wo viele nominale Ausdrucksvarianten der Prädikation möglich sind.

Der zusammengesetzte Satz kann sowohl parataktisch als auch hypotaktisch gebaut sein. Wichtig dabei ist zu wissen, dass auch Hauptsätze nicht immer von Nebensätzen unabhängig sind und also nicht immer allein stehen können. Vom zusammengesetzten Satz ist der zusammengezogene Satz zu unterscheiden, der seinerseits nicht identisch mit der Aufzählung ist.

Die Ellipse ist eine unvollständige Satzkonstruktion, die im Prinzip auf einem Verstoß gegen das syntaktische Minimum beruht, jedoch rezeptiv akzeptabel ist, weil sie eine kommunikative Funktion erfüllt.

Ein Pendant zur Ellipse ist in quantitativer Hinsicht die Satzperiode, die ihrerseits so komplex gebaut ist, dass sie Verständigungsschwierigkeiten verursachen kann und daher auf die Anwendung in gewissen Funktionalstilen beschränkt ist (z. B. philosophische oder literarische Texte).

Die Verbvalenz prädestiniert die Grundstruktur und damit die Form eines Satzes. Fakultative Aktanten können fehlen, wenn deren informativer Beitrag im gemeinsamen Wissen der Kommunikationspartner verankert und daher nachvollziehbar ist. Das unterscheidet sie von den freien Angaben, die beliebig hinzugefügt werden können und daher auch als latente Sätze zu verstehen sind.

Die Verbvalenz ist im Prinzip eine semantisch geprägte Kategorie, da die Verbsememe konstituierenden Seme die Anzahl der notwendigen Aktanten bestimmen. Die meisten Verben sind polysem. Deshalb muss ihre Valenz und damit die potentiell mögliche Formalisierung auf der Oberfläche für jedes Semem gesondert beachtet werden. 
Die Verbvalenz kann auch modifiziert werden. Meistens geht es um eine Art Valenzminderung (Valenzreduktion). In diesem Fall sind auch verkürzte Satzformen ohne obligatorische Aktanten grammatisch richtig und verständlich, wenn pragmatisches Wissen von Kommunikationspartnern aktiviert werden kann.

\section{Literatur}

BRAČIČ, Stojan/Ulla, FIX/Albrecht, GREULE (2018) Textgrammatik, Textsemantik, Textstilistik: ein textlinguistisches Repetitorium. 2., bearbeitete und erweiterte Auflage. Ljubljana: Znanstvena založba Filozofske fakultete.

EROMS, Hans-Werner (2000) Syntax der deutschen Sprache. Berlin/New York: de Gruyter.

FANDRYCH, Christian/Maria, THURMAIR (2018) Grammatik im Fach Deutsch als Fremd- und Zweitsprache: Grundlagen und Vermittlung. Grundlagen Deutsch als Fremd- und Zweitsprache 2. Berlin: Erich Schmidt Verlag.

FIX, Ulla (1992) „Stilanalyse - ein Mittel der Erziehung zum Widerspruch? Pragmastilistische Analyse eines Anweisungstextes." Deutschunterricht 45, 3. 128-136.

FIX, Ulla (2018) „Textstilistik.“ In: S. Bračič/U. Fix/A. Greule, 111-143.

GREULE, Albrecht (2018) „Textgrammatik.“ In: S. Bračič/U. Fix/A. Greule, 5-15. HELBIG, Gerhard/Joachim, BUSCHA (1998) Deutsche Grammatik. Ein Handbuch für den Ausländerunterricht. Leipzig/München: Langenscheidt.

HELBIG, Gerhard/Wolfgang, SCHENKEL (1982) Wörterbuch zur Valenz und Distribution deutscher Verben. Leipzig: VEB Bibliographisches Institut Leipzig.

JUNG, Walter (1990) Grammatik der deutschen Sprache. Mannheim/Leipzig: Bibliographisches Institut.

KREVS BIRK, Uršula (2006) Argumentellipse. Aktantenweglassung in deutschen und slowenischen Reportagetexten. Tübingen: Gunter Narr Verlag.

MASCOLO, Georg (2010) „Der Krieg 1939-1945. Als die Welt in Flammen stand.“ Der Spiegel Geschichte 3, 80.

WÖLLSTEIN, Angelika et al. (Hrsg.) (2016) Duden: Die Grammatik. Unentbehrlich für richtiges Deutsch. 9., völlig überarbeitete und aktualisierte Auflage. Berlin: Dudenverlag.

\section{Zusammenfassung \\ ZU DEN SATZFORMEN IM DEUTSCHEN UND WIE SIE DURCH DIE VERB- VALENZ BEEINFLUSST WERDEN}

Im Ganzsatz ist das Kriterium für die Bestimmung der Satzform die Anzahl der explizit ausgedrückten Prädikationen. Der Ganzsatz ist dabei ein Oberbegriff, der für alle Satzkonstruktionen stehen kann, deren Erkennungsmerkmale die Majuskel am Anfang und ein Interpunktionszeichen am Ende sind, und somit sowohl Einfachsätze als auch komplexe Sätze umfasst. Der zusammengesetzte Satz kann sowohl parataktisch als auch hypotaktisch gebaut sein. Wichtig dabei ist zu wissen, dass auch Hauptsätze 
nicht immer von Nebensätzen unabhängig sind und also nicht immer ohne diese stehen können. Durch die Verbvalenz als eine semantisch geprägte Kategorie sind die Grundstruktur und damit die Form eines Satzes prädestiniert. Fakultative Aktanten können fehlen, wenn deren informativer Beitrag im gemeinsamen Wissen der Kommunikationspartner kotextuell oder situativ verankert und daher nachvollziehbar ist. Die Verbvalenz kann auch modifiziert werden. Meistens geht es um eine Art Valenzminderung (Valenzreduktion). In diesem Fall sind auch verkürzte Satzformen ohne obligatorische Aktanten grammatisch richtig und verständlich, soweit pragmatisches Wissen von Kommunikationspartnern aktiviert werden kann. Die Ellipse ist eine unvollständige Satzkonstruktion, die im Prinzip auf einem Verstoß gegen das syntaktische Minimum beruht, jedoch rezeptiv akzeptabel ist, weil sie eine kommunikative Funktion erfüllt.

Schlüsselwörter: Syntax, Satzformen, Verbvalenz, pragmatisches Wissen, Ellipse

\section{Abstract \\ ON SENTENCE FORMS IN GERMAN AND ON THE INFLUENCE OF VALENCY ON THEM}

The criterion for the classification of the German sentence forms is the number of explicitly expressed (verbal) statements. The sentence is to be understood as a hyperonym for all constructions between the capital letter at the beginning and the punctuation mark at the end; it comprises both clauses and sentences. The sentence can be coordinate or subordinate. Importantly, the main clause is not always independent from the subordinate clause (subclause) and it cannot always be used on its own. The verb valency is a semantically determined category that influences the basic structure as well as the form of the sentence. Non-obligatory agents can be omitted if their information value is part of the communicative partners' co-text or context-of-the-situation knowledge and can easily be reconstructed. The verb valency can also be modified, in most cases in the form of valency reduction. Such reductions are grammatically correct and understandable without obligatory complements provided that the pragmatic knowledge of the participants in the communicative process can be activated. Another type of valency reduction is an incomplete sentence construction, the so-called ellipsis. It results from a deliberate breach of the rule of syntactic minimum. It is acceptable to the recepient as long as it fulfills a communicative function.

Keywords: syntax, forms of sentences, verb valency, pragmatic knowledge, ellipsis 


\section{Povzetek \\ K OBLIKAM NEMŠKE POVEDI IN VPLIVU VEZLJIVOSTI}

Merilo za določitev oblike nemške povedi je število eksplicitno izraženih predikacij. Poved je pri tem nadpomenka, ki stoji za vse stavčne konstrukcije, katerih razpoznavna znaka sta velika začetnica na začetku in ločilo na koncu, in tako zaobjema tako proste kakor tudi zložene stavke. Zložena poved je lahko zgrajena tako parataktično kakor tudi hipotaktično. Pri tem je pomembno vedeti, da tudi glavni stavki niso vedno neodvisni od odvisnikov in da torej ne morejo vedno stati samostojno. Glagolska vezljivost kot semantično pogojena kategorija vnaprej določa temeljno strukturo in s tem obliko povedi. Fakultativni aktanti/delovalniki/udeleženci lahko manjkajo, če je njihov informativni prispevek sobesedilno ali sotvarno vtkan v skupno znanje sporazumevalnih partnerjev in ga je na ta način mogoče priklicati v zavest. Glagolska vezljivost je lahko tudi modificirana. Največkrat gre za vrsto valenčne redukcije. V tem primeru so slovnično pravilne in razumljive tudi skrajšane strukture povedi brez obveznih delovalnikov, pod pogojem, da je mogoče aktivirati pragmatično védenje udeležencev sporazumevalnega procesa. Izpust je nepopolna stavčna konstrukcija, ki načeloma sloni na neupoštevanju skladenjskega minimuma, vendar je z vidika naslovnika sprejemljiva, ker izpolnjuje neko komunikativno funkcijo.

Ključne besede: skladnja, oblike povedi, glagolska vezljivost, pragmatično védenje, izpust 